prolonged faecal tagging). We evaluated the diagnostic accuracy of FIT for colorectal cancer (CRC) and other significant bowel disease (SBD: inflammatory bowel disease, high-risk polyps as per 2020 BSG post-polypectomy guidelines) across a range of FIT thresholds $(<10 \mu \mathrm{g} / \mathrm{g}, 10-50 \mu \mathrm{g} / \mathrm{g}$, and $>50 \mu \mathrm{g} / \mathrm{g})$. Multivariable logistic regression was performed to assess the predictive value of various demographic (age, gender) and clinical variables (lower GI symptoms, two-week vs non twoweek referrals) in the prediction of CRC and SBD.

Results 481 patients (21-95 years, median 62 years) were included in the analysis. The prevalence of CRC in this cohort was $6.6 \%$. The negative predictive values of FIT for CRC at three cut-offs $(10 \mu \mathrm{g} / \mathrm{g}, 50 \mu \mathrm{g} / \mathrm{g}$ and $100 \mu \mathrm{g} / \mathrm{g})$ were 98.3\% (95\%CI 93.6-99.5\%), 98.1\% (95\%CI 96.2-99.1\%) and $96.7 \% \quad(95 \% \mathrm{CI} 94.9-97.9)$ respectively. The sensitivities at these cut-offs were $93.8 \%$ (95\%CI 79.2-99.2\%), 81.3\% (95\% CI $63.6-92.8 \%)$ and $62.5 \%$ (95\%CI $43.7-78.9 \%)$ respectively. Based on a $10 \mu \mathrm{g} / \mathrm{g}$ cut-off, two CRCs would have been missed, both of which arose in males over 75 years old. Multivariate regression analyses demonstrate that increasing age, male gender, and FIT $>50 \mu \mathrm{g} / \mathrm{g}$ are the sole of predictors of CRC (see table) and SBD.

Conclusions A combination of age, male gender and FIT values are most predictive variables of future CRC and SBD risk. In the current post-COVID environment, FIT can play a role in streamlining lower GI investigations for two-week referrals, where FIT $>50 \mu \mathrm{g} / \mathrm{g}$ are prioritised as most urgent. Units with significant endoscopy backlogs could also consider de-escalating two-week referrals of younger patients with low FIT values $(<10 \mu \mathrm{g} / \mathrm{g})$.

\section{PTH-109 THE BOTTOM LINE: REAL WORLD MANAGEMENT OF ACUTE LOWER GASTROINTESTINAL BLEEDING COMPARED TO BSG GUIDELINES}

${ }^{1}$ Mike Davies*, ${ }^{2}$ Tristan Townsend, ${ }^{3}$ Matthew Dixon, ${ }^{4}$ Violeta Razanskaite, ${ }^{3}$ James Morgan, ${ }^{5}$ Daniyal Baig, ${ }^{6}$ James Fox, ${ }^{6}$ Vinay Kumar, ${ }^{4}$ Nada Elamin, ${ }^{4}$ lannis Papmargaritas, ${ }^{2}$ Doug Penman, ${ }^{2}$ Thomas Conley, ${ }^{2}$ Joseph Fiske, ${ }^{3}$ Kieran Walker, ${ }^{7}$ Waqas Gaba, ${ }^{2}$ Sreedhar Subramanian, ${ }^{2}$ Philip I Smith. ${ }^{1}$ Countess of Chester Hospital, Chester, UK; ${ }^{2}$ Royal Liverpool University Hospital, Liverpool, UK; ${ }^{3}$ Arrowe Park Hospital, UK; ${ }^{4}$ Aintree University Hospital, Liverpool, UK; ${ }^{5}$ Whiston Hospital, UK; ${ }^{6}$ Macclesfield District General Hospital, Macclesfield, UK; ${ }^{7}$ Southport Hospital, Southport, UK

\subsection{6/gutjnl-2021-BSG.312}

Introduction Lower gastrointestinal bleeding (LGIB) is a common hospital presentation, from self-limiting per-rectal bleeding to a life-threatening haemorrhage. British Society of Gastroenterology (BSG) acute LGIB guidelines define a clear management approach including risk stratification for patient management. However, real-world management of LGIB in relation to this guidance is currently unknown.

Methods Patients aged $\geq 16$ years presenting with LGIB to 7 hospital trusts from June 1st-Aug 31st 2019 were included. Data on presentation, management and outcomes of patients were recorded. These were audited against BSG guidelines.

Results 407 patients were included. 51\% were male with a mean age of $60(\mathrm{SD}=22)$. Mean Shock Index (SI) at presentation was 0.69 , with a SI $\geq 1$ being rare (6.3\%). 2.2\% (9/ 407 ) of patients remained haemodynamically unstable (SI >1) after initial resuscitation. Of these, 22.2\% underwent a computed tomogram angiography (CTA). Within the major bleed risk patients (Oakland Score >8); 284 (85\%) were admitted and $50(15 \%)$ were discharged from A\&E. For minor bleed risk patients (Oakland Score $\leq 8$ ); 67.9\% and 32.1\% were admitted and discharged respectively. Complete Oakland Score data was unavailable for 7 patients. Of admitted patients, colonoscopy and sigmoidoscopy was performed in $4.3 \%$ and $14.6 \%$ respectively, whilst $81.8 \%$ underwent no inpatient LGI endoscopy. A bleeding site was seen in 12 (20\%) patients at endoscopy, for which $2(10 \%)$ received endoscopic therapy. 7 day rebleeding rates were higher in patients who underwent LGI endoscopy versus those conservatively managed $(16.7 \%$ vs $7.5 \%, \mathrm{p}=0.028)$. Inpatient mortality was low at $2.1 \%$, with no difference in major vs minor bleed patients $(2.1 \%$ vs $2.6 \%, \mathrm{p}=1.0$ ). Median length of stay was 5.5 days in patients who received LGI endoscopy and 2 days for those conservatively managed $(p=<0.00001) .15 .3 \%$ of patients were managed in accordance with BSG guidance. The most common deviations being patients with an Oakland Score $>8$ being discharged and admitted patients not undergoing LGI endoscopy. Conclusions Real world practice of managing patients presenting with LGIB is not in keeping with current BSG guidelines, with admission or discharge often not in keeping with Oakland Scores. The majority of admitted patients do not receive inpatient LGI endoscopy, in patients who do, endoscopic therapy is rarely indicated.

\section{PTH-110 AN AUDIT EVALUATING REPORTING OF PT1 COLORECTAL CANCERS AT THE ROYAL DEVON AND EXETER HOSPITAL}

Elinor George*, Trupti Mandalia. Royal Devon and Exeter NHS Foundation Trust, Exeter, UK

\subsection{6/gutjnl-2021-BSG.313}

Introduction The pathology report plays a vital role in assisting the multidisciplinary team to risk stratify colorectal cancers (CRCs). We evaluated our departmental practice of reporting polyp cancers in local resection specimens and suggested appropriate recommendations for quality improvement.

Methods A database search identified patients who had been diagnosed with pT1 CRCs in colonic polyps between March 2014 and December 2018. Gold standards for histologic reporting of specimens were obtained from the Royal College of Pathologists' minimum data set and NHS Bowel Cancer Screening Programme (NHSBCSP) guidelines. An internal review of pathology reports assessed compliance against these gold standards and the inclusion of newly emerged prognostic parameters.

Results 70 patients diagnosed with pT1 CRC in colonic polyps were identified during this period. $32.8 \%$ of these cases were polyps identified through the Bowel Cancer Screening programme. Two pathologists, as recommended by the NHSBCSP, reported $100 \%$ of cases. Tumour differentiation and lymphovascular invasion were reported in over $90 \%$ and $80 \%$ of cases. $100 \%$ of sessile polyps were assigned a Kikuchi level and/or carried a comment to that effect. Haggit level was reported in $86 \%$ of pedunculated polyps. In $17 \%$ of cases, no comment on the level of submucosal invasion was included. In the majority of these cases (75\%), it was deemed difficult to classify the polyps as either pedunculated or sessile, likely due to fragmentation or suboptimal orientation. The depth and width of the invasive tumour, as described by Ueno et al. ${ }^{1}$, were reported in $47 \%$ and $41 \%$ respectively. $20 \%$ of reports did not mention the distance from the deep margin, $12.8 \%$ of the $20 \%$ stated this margin was not assessable. The 